\title{
Thorough QT Study
}

National Cancer Institute

\section{Source}

National Cancer Institute. Thorough QT Study. NCI Thesaurus. Code C158287.

A type of study designed to evaluate the potential for an experimental drug to delay cardiac ventricular repolarization. 